\title{
CircMTO1 inhibits ox-LDL-stimulated vascular smooth muscle cell proliferation and migration via regulating the miR-182-5p/ RASA1 axis
}

\author{
Ningning Ji, Yu Wang, Xinyan Gong, Shimao Ni and Hui Zhang ${ }^{*}$ (])
}

\begin{abstract}
Background: Circular RNAs (circRNAs) play critical roles in the development of atherosclerosis (AS). This study investigated the role of circMTO1 in the progression of AS.

Methods: Serum samples from AS patients and healthy volunteers and vascular smooth muscle cells (VSMCs) were used as the study materials. The expressions of circMTO1 and miR-182-5p were measured by RT-qPCR. The effects of circMTO1, miR-182-5p, and RASA1 on VSMC proliferation and apoptosis were examined by MTT and BrdU assays and wound healing and flow cytometric analyses, respectively. Downstream target genes of circMTO1 and miR-182-5p were predicted using target gene prediction and screening and confirmed using a luciferase reporter assay. RASA1 expression was detected by RT-qPCR and Western blot.
\end{abstract}

Results: circMTO1 expression was decreased, while miR-182-5p expression was increased in human AS sera and oxidized low-density lipoprotein (ox-LDL)-stimulated VSMCs. CircMTO1 overexpression inhibited the proliferation and promoted the apoptosis of ox-LDL-stimulated VSMCs. CircMTO1 was found to be served as a sponge of miR-182-5p and RASA1 as a target of miR-182-5p. Moreover, circMTO1 acted as a ceRNA of miR-182-5p to enhance RASA1 expression. Furthermore, miR-182-5p overexpression and RASA1 knockdown reversed the effects of circMTO1 overexpression on the proliferation, migration, and apoptosis of ox-LDL-stimulated VSMCs.

Conclusion: CircMTO1 inhibited the proliferation and promoted the apoptosis of ox-LDL-stimulated VSMCs by regulating miR-182-5p/RASA1 axis. These results suggest that circMTO1 has potential in AS treatment.

Keywords: CircMTO1, miR-182-5p, RASA1, VSMCs, Proliferation, Apoptosis

\section{Background}

Arteriosclerosis (AS) has become one of the major causes of increased mortality and incidence rates of cardiovascular and cerebrovascular diseases worldwide (Lippincott and Wilkins 2017; Tabib et al. 2017). It has seriously affected the quality of life of the middle-aged and the

*Correspondence: huizhang03@aliyun.com

Department of Cardiology, Yiwu Central Hospital, Affiliated Hospital of Wenzhou Medical University, No.699, Jiangdong Road, Yiwu

City 322000, Zhejiang Province, People's Republic of China elderly and increased the social burden (Asayama et al. 2017). The leading pathological cause and characteristic of this chronic inflammatory disease is the hardening of the arterial wall and narrowing of the lumen caused by atherosclerotic plaques (Tölle et al. 2015). Lower extremity arteriosclerosis obliterans (ASO) is the most common occlusive disease of peripheral blood vessels, and AS can cause ASO. The pathogenesis of AS has been extensively studied and explored. At present, the mechanism of activation of vascular smooth muscle cells (VSMCs) has original author(s) and the source, provide a link to the Creative Commons licence, and indicate if changes were made. The images or other third party material in this article are included in the article's Creative Commons licence, unless indicated otherwise in a credit line to the material. If material is not included in the article's Creative Commons licence and your intended use is not permitted by statutory regulation or exceeds the permitted use, you will need to obtain permission directly from the copyright holder. To view a copy of this licence, visit http://creativecommons.org/licenses/by/4.0/. 
gradually attracted scholars' attention (Zhao et al. 2014; Begum et al. 2011). In the early stages of the disease, the main feature is that abnormal proliferation of VSMCs leads to intimal thickening and lumen shrinkage (Rotllan et al. 2015). It is well known that the proliferation of VSMCs is the common pathogenesis of cardiovascular and cerebrovascular diseases. Due to the limitations in the choice of clinical treatment and the prognosis effect (Zhang et al. 2011), more and more scholars have begun to pay attention to the pathogenesis of arteriosclerosis, hoping to interfere or block the occurrence of arteriosclerosis.

Oxidized low-density lipoprotein (ox-LDL) has been proven to be involved in the formation and progression of atherosclerotic plaque (Li et al. 2017). Studies have shown that a low concentration of ox-LDL can promote the proliferation of VSMCs, while a high concentration of ox-LDL can promote the apoptosis of VSMCs (Wang et al. 2019a). Therefore, in this study, ox-LDL was used to establish the proliferation model of VSMCs to explore the mechanism of atherosclerosis further.

Many basic studies in recent years have confirmed that the expression profiles of many genes are often changed in the pathophysiology of cardiovascular diseases (Wallace et al. 2008). In recent studies, it has been reported that circular RNA (circRNA) is involved in the pathophysiological process of cardiovascular disease, diabetes, and cancer (Gruner et al. 2016; Ashwal-Fluss et al. 2014). More and more basic research results have shown that circRNA plays a critical role in the occurrence and progression of cardiovascular diseases (Fan et al. 2017; Holdt et al. 2018). For example, studies have found that multiple circRNAs act as potential biomarkers in coronary atherosclerosis. A variety of circRNAs in circulating blood are beneficial to the screening and early diagnosis of coronary atherosclerotic heart disease (Qu et al. 2017). CircMTO1 is a type of circRNA which is conserved in mouse and human (Additional file 1). It has been found that circMTO1 is abnormally expressed in many diseases, such as liver cancer and glioblastoma (Han et al. 2017a). However, it is unclear whether circMTO1 is involved in the proliferation of VSMCs.

Numerous studies have shown that circRNAs regulate gene expression by acting as miRNA sponges to competitively bind to messenger RNAs (mRNAs) and proteins to form a complex, regulating mRNA splicing, translation, and degradation (Jin et al. 2016). More than 2000 miRNAs have been discovered in the human genome (Anokyedanso et al. 2011). MiRNAs play essential roles in the development of tumors, endocrine diseases, nervous system diseases, and cardiovascular diseases, to name a few (Chen et al. 2017; Piwecka et al. 2017). Recent studies have found that miRNAs participate in controlling
VSMCs' proliferation. MiR-132, miR-22, miR-379 and miR-124 inhibit, while miR-214, miR-221 and miR-146 promote VSMCs' proliferation (Guo et al. 2016a; Li et al. 2018). Previous studies have shown that miR-182-5p is involved in cancer, myocardial ischemia and reperfusioninduced injuries, and spinal neuronal diseases ( $\mathrm{Li}$ et al. 2014; Xue et al. 2016). However, whether miR-182-5p is involved in VSMCs' proliferation is not fully understood. RASA1 is a cytoplasmic protein with a molecular weight of $120 \mathrm{kD}$. Its C-terminus is a GTPase-activated protein (GAP) domain with catalytic activity (Gong et al. 2015). Its $\mathrm{N}$-terminus consists of $\mathrm{PH}, \mathrm{CaLB} / \mathrm{C} 2, \mathrm{SH} 2$, and SH3 domains. RASA1 activates Ras GTPase, which hydrolyzes Ras GTP and inhibits Ras signaling pathway. Studies have shown that RASA1 promotes cell apoptosis by inhibiting the Ras-MAPK pathway, which is closely related to various diseases (Revencu et al. 2013; Hancock et al. 2014). Therefore, we hypothesized that circMTO1 inhibits VSMCs' proliferation by modulating miR-182-5p/RASA1 axis. The study's main purpose was to investigate the mechanism by which circMTO1 regulates VSMCs' proliferation with the hope to provide new ideas for the diagnosis and targeted therapy of AS and a theoretical basis and experimental evidence for elucidating the mechanism of circRNA-related vascular lesions and finding potential therapeutic targets.

\section{Materials and methods}

\section{Clinical samples}

This study was conducted with the approval of the Yiwu Central Hospital's Ethics Committee, and all participants signed the informed consent (50-70 years old, 40\% female). Blood samples were collected from AS patients $(\mathrm{n}=55)$ and healthy volunteers $(\mathrm{n}=55)$ in centrifuge tubes without anticoagulants. Serum was extracted by centrifugation for RNA isolation using TRIzol LS reagent (Yanjin, Shanghai, China). None of the healthy volunteers had AS disease, malignant tumors, and inflammatory diseases $(<1$ month $)$.

\section{Cell culture}

Human aortic vascular smooth muscle cells (HA-VSMC) were obtained from the Fenghui Biotechnology Co., Ltd (Hunan, Shanghai, China) and maintained in F-12 K medium supplemented with 10\% FBS (Invitrogen) at $37^{\circ} \mathrm{C}$ in a humidified atmosphere containing $5 \% \mathrm{CO}_{2}$.

\section{Cell transfection and treatment}

The full-length of circMTO1 was amplified by PCR and cloned into pcDNA3.1 vector (Invitrogen) to construct a pcDNA-circMTO1 overexpression plasmid. Si-circMTO1 and its control si-NC, si-RASA1 and its control si-NC, and miR-182-5p mimic (miR-182-5p) and its 
control miR-NC were all designed and synthesized by GenePharma (Suzhou, China). VSMCs were treated with ox-LDL (Luwen, Shanghai, China) to detect the expression of circMTO1 and miR-182-5p. CircMTO1, miR182-5p, and RASA1 sequences are provided in Additional file 1.

\section{qRT-PCR}

Total RNA in cells was extracted using TRIzol LS reagent (for serum RNA extraction) (Yanjin, Shanghai, China). microRNA was extracted and purified using TaqMan ABC miRNA Purification Kit (Thermo Fisher Scientific, USA). After the reverse transcription reaction, qRT-PCR was performed using a ViiATM 7 real-time PCR system (Life Technologies, Grand Island, NY). The miR-182-5p level was normalized to U6, and circMTO1, MTO1 and RASA1 were normalized to GAPDH. The relative expression levels of each gene were calculated and normalized using the $2^{-\Delta \Delta C t}$ method. The specific qRT-PCR for circMTO1, MTO1, and GAPDH were performed as previously reported (Li et al. 2013) with the following primers: CircMTO1 (divergent primer): forward 5'-GGCCAT CCTATGTCAGTTG-3'; CircMTO1 (divergent primer) reverse 5'-AGGTAGGCCCGCACGGT-3'; MTO1 (convergent primer) forward: 5'-AAGTG CCGTTGGGT GTGG-3'; MTO1 (convergent primer) reverse: 5'-CAA TCATTCGTT GGAGGTT-3'; MiR-182-5p forward: 5'-CCCAACT GTATGGTTT-3'; MiR-182-5p reverse: 5'-CGGATGGCCCAACGG-3'; RASA1 forward: 5'-TCG AAAAGCTATGC TATGGC-3'; RASA1 reverse: $5^{\prime}$-CTA ACAATCACGTGCGCGA-3'; GAPDH forward: 5'-CGA GAGAGCGATCAGACCT-3'; GAPDH reverse: 5'-GTA TAGTTGCT CACGGGAAC-3'; U6 forward: 5'-ATG TGGTATGACACCTGGGCC- $3^{\prime}$ and U6 reverse: $5^{\prime}$-GAT TGGCAGCGATTATACACC-3'.

\section{Cell proliferation assay}

For the CCK- 8 assay, transfected cells were seeded into 96-well plates at a density of 2000 cells per well. After $48 \mathrm{~h}$, cell viability was measured by the Cell Counting Kit-8 (CCK-8) system (Liji, Shanghai, China). The absorbance at $450 \mathrm{~nm}$ of each well was measured using a microplate reader (Tecan, Switzerland). For the BrdU incorporation assays, transfected cells were seeded in 96-well plates at a density of 2000 cells per well. After $48 \mathrm{~h}$, cell proliferation was analyzed using the BrdU Cell Proliferation Assay Kit (\#5213S, Cell Signaling).

\section{Cell apoptosis assay}

The transfected cells were collected, washed, and incubated with $500 \mu \mathrm{l}$ of binding buffer, $5 \mu \mathrm{l}$ of FITC Annexin $\mathrm{V}$, and $5 \mu \mathrm{l}$ of propidium iodide (PI). The apoptotic rate was determined using flow cytometry (FACS Calibur, USA) following the manufacturer's instructions.

\section{Dual-luciferase reporter gene assay}

The wild-type or mutant sequences of the circMTO1 or RASA1 3'-untranslated region (3'-UTR) were cloned into the pmirGLO reporter plasmids. VSMCs were co-transfected with pcDNA3.1, pcDNA-circMTO1 (wild type or mutant), the negative control, or miR-182-5p. After $48 \mathrm{~h}$ of transfection, luciferase activity was measured using a dual-luciferase reporter assay system (Promega).

\section{Wound healing assay}

$24 \mathrm{~h}$ before transfection, cells were digested and passaged to 12-well plates. The cell confluency at transfection was $60-80 \%$. The experimental group and the control group were set up parallelly. The transfection concentration was $50 \mathrm{nmol} / \mathrm{L}$. $24 \mathrm{~h}$ after transfection, a tip was used to draw a cell-free area of substantially the same width in each well to mimic wounds. Capecitabine (Abcam, USA), as the DNA replication inhibitor, was added into the culture media at a final concentration of $12 \mathrm{nM}$. The cells were then cultured for $24 \mathrm{~h}$ to observe their ability to migrate.

\section{Western blot}

The transfected cells were collected, and total proteins were extracted and quantified using the BCA Protein Assay Kit. After separated and transferred onto PVDF membranes, proteins were incubated with antibodies against RASA1 (1:500; Abcam, ab40677), a-SMA (1:500; Abcam, ab5694), LDL-receptor (1:1000; Abcam, ab52818), caspase 3 (1:1000; Abcam, ab32315), cyclin D1 (1:1000; Abcam, ab16663) and GAPDH (1:1000; Sangon, Shanghai), overnight at $4{ }^{\circ} \mathrm{C}$ respectively. After washed, the proteins were incubated with 1:2000 HRP-labeled anti-rabbit secondary antibody (Catalogue No. ab150077) for $1 \mathrm{~h}$ and visualized using ECL reagent. Western blot analyses were performed as previously reported (Kuhar and Yoho 2015).

\section{AS animal model}

A total of 15 10-week-old male ApoE ${ }^{-/-}$C57BL/6 mice from the Animal Center of Shanghai (Shanghai Lab. Animal Research Center) were used in the study. These mice were fed on a Western-type diet (contain $21 \%$ fat by weight, $0.15 \%$ cholesterol) for 5 weeks to generate atherosclerotic plaques (Wang et al. 2019b), AAV-pcDNA, AAV-pcDNA-circMTO1, and AAV-pcDNA-circMTO1/ AAV-miR-182-5p were injected into these mice via tail veins $\left(1 \times 10^{11} \mathrm{PFU} / \mathrm{ml}, 1 \mu \mathrm{L}\right)$ once every two weeks, respectively, mice were then fed on a chow diet for the next 30 weeks, and sacrificed. Aortas from the base at 
the aortic valve up to the diaphragm were collected as described previously (Paigen et al. 1987) (Table 1).

\section{Histological evaluation}

After the AS model was established, the whole aorta from the heart to the abdominal aorta was dissected. Atherosclerotic plaques in the aortas were stained with $0.5 \%$ oil-red O (Sigma-Aldrich) for $15 \mathrm{~min}$ at room temperature. Serial cryosections $(8 \mu \mathrm{m})$ were cut along the aortic root specimens at $-20^{\circ} \mathrm{C}$ using a cryotome (HM550, Thermo Scientific, USA). The sections were tained with hematoxylin/eosin and oil-red $\mathrm{O}$ and counterstained with DAPI to evaluate the lipid content in the plaque areas.

\section{Immunochemistry}

OCT-embedded myocardial tissue sections were used for immunochemistry. Serial sections of the aortic root beyond the end of the aortic sinus were selected for staining. Rabbit obtained antibodies for $\alpha$-actin (Abcam, $0.5 \mu \mathrm{g} / \mathrm{ml}$ ) was chosen for probing the smooth muscle and foam cells. Goat anti-rabbit antibodies were also used as secondary antibodies. The staining images were captured using a Leica microscope.

\section{Statistical method}

The monitoring data were analyzed by SPSS19.0 statistical software. Results of the data analysis were shown as mean \pm standard deviation (mean $\pm \mathrm{SD}$ ). Multigroup data analysis was based on one-way ANOVA followed by the LSD test. $\mathrm{P}<0.05$ indicated a significant difference.

\section{Results}

\section{CircMTO1 expression was reduced in the serum of AS patients and ox-LDL-stimulated VSMCs}

Firstly, circMTO1 expression in serum of AS patients and ox-LDL-stimulated VSMCs was examined. As shown in Fig. 1A, circMTO1 expression was significantly lower in AS patients $(n=55)$ than that in the health group $(n=55)$ $(\mathrm{P}<0.01)$. Moreover, there was no significant difference in MTO1 mRNA expression $(P>0.05)$ (Fig. 1B). As shown in Fig. $1 \mathrm{C}$ and $\mathrm{D}$, with ox-LDL concentration increasing and action time prolonging, circMTO1 expression in VSMCs gradually decreased $(\mathrm{P}<0.01)$, while no significant difference was found in MTO1 mRNA expression. Based on the results, $50 \mu \mathrm{g} / \mathrm{ml}$ ox-LDL treatment for $48 \mathrm{~h}$ was selected for subsequent experiments. These results demonstrate that circMTO1 plays a role in the progression of AS.

\section{CircMTO1 overexpression inhibited proliferation and migration of ox-LDL-stimulated VSMCs}

To further analyze the role of circMTO1 in ox-LDLstimulated VSMCs, pcDNA 3.1-circMTO1 overexpression plasmid was transfected into VSMCs. As shown in Fig. 2, compared with the $\mathrm{NC}$ group, the expression of circMTO1 in the circMTO1 overexpression group was significantly increased (Fig. 2A, $\mathrm{P}<0.01$ ); ox-LDL stimulation significantly increased proliferation, but reduced apoptosis of VSMCs (Fig. 2B, $\mathrm{P}<0.01$ ). Interestingly, circMTO1 overexpression effectively attenuated oxLDL-stimulated cell proliferation, promoted ox-LDLstimulated cell apoptosis (Fig. 2D, $\mathrm{P}<0.01$ ), and inhibited ox-LDL-stimulated cell migration (Fig. $2 \mathrm{E}, \mathrm{P}<0.01$ ). The above results indicated that circMTO1 overexpression inhibited the proliferation and migration of ox-LDLstimulated VSMCs and induced apoptosis of ox-LDLstimulated VSMCs.

\section{CircMTO1 served as a sponge of miR-182-5p}

Using the network tool Starbase, we predicted that circMTO1 might bind to miR-182-5p (Fig. 3A). As shown in Fig. 3B, we found that miR-182-5p overexpression significantly reduced the luciferase activity of wild-type $3^{\prime}$-UTR of circMTO1 $(\mathrm{P}<0.01)$, but not the mutated $3^{\prime}$-UTR of circMTO1. Furthermore, as shown in Fig. 3C, compared with the NC group, circMTO1 knockdown significantly increased miR-182-5p expression in VSMCs, while circMTO1 overexpression significantly reduced miR-182-5p expression in VSMCs $(\mathrm{P}<0.01)$. In addition, compared with the healthy group, serum miR-182-5p expression was significantly increased in AS patients $(\mathrm{P}<0.01)$ (Fig. 3D), and there was a strong negative correlation $(\mathrm{r}=-0.588, \mathrm{P}<0.001)$ between the expression of circMTO1 and miR-182-5p in AS patients (Fig. 3E). These results indicated that circMTO1 might exert its biological function through miR-182-5p sponging.

Table 1 Antibodies list

\begin{tabular}{|c|c|c|c|c|}
\hline Name & Reactivity species & Application & Company & Cat. .No \\
\hline RASA antibody & Mouse, human & WB & Abcam & Ab40677 \\
\hline a-SMA antibody & Mouse, human & $\mathrm{HC}, \mathrm{WB}$ & Abcam & Ab7817 \\
\hline LDL-receptor antibody & Mouse, human & WB & Abcam & Ab30532 \\
\hline Caspase3 antibody & Mouse, human & WB & Abcam & Ab13847 \\
\hline Cyclin D1 antibody & Mouse, human & WB & Abcam & Ab16663 \\
\hline
\end{tabular}



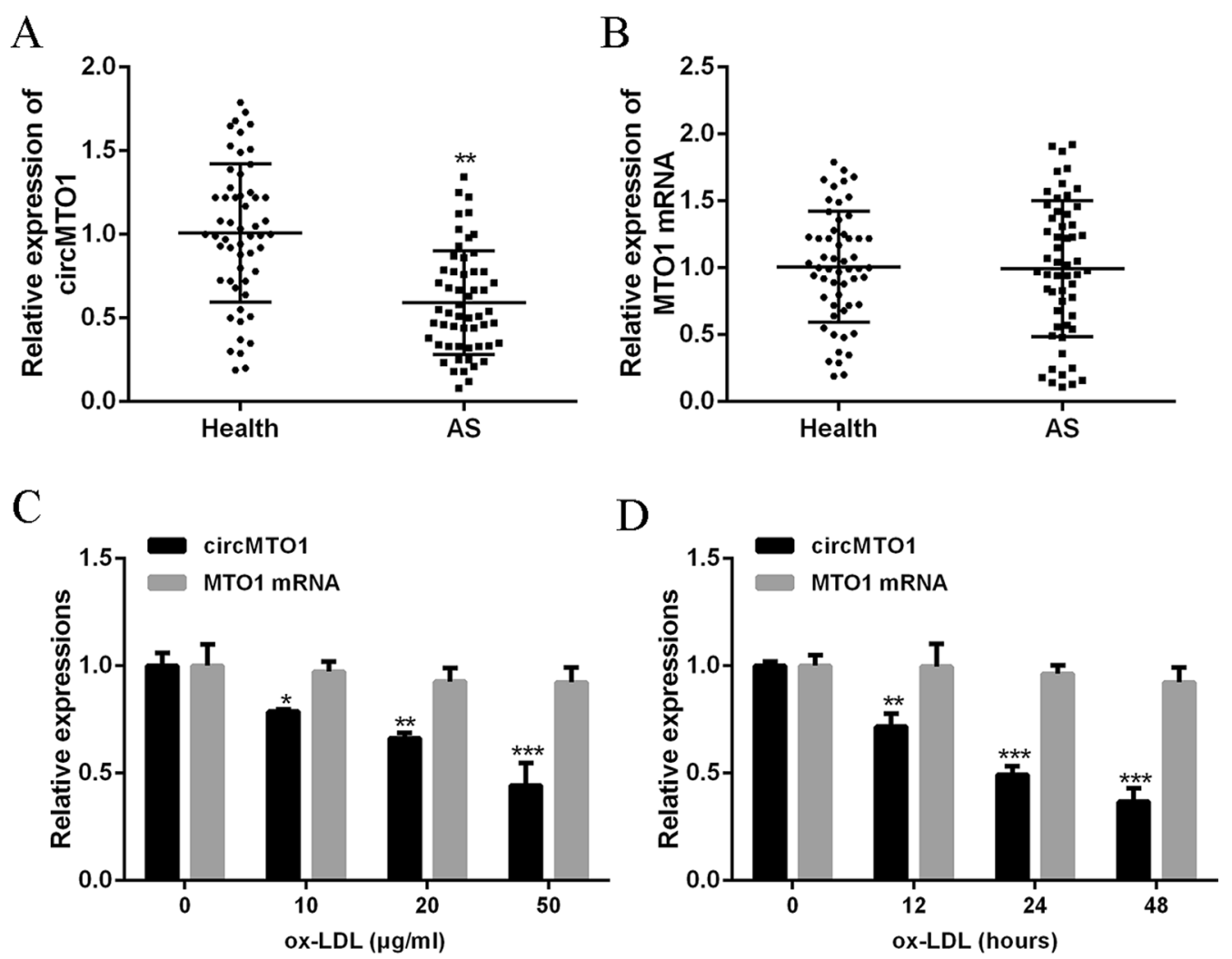

Fig. 1 Reduced circMTO1 expression in serum and ox-LDL-stimulated VSMCS. A, B The mRNA levels of circMTO1 and MTO1 in serum of AS patients $(n=55)$ and healthy volunteers $(n=55)$. C The mRNA levels of circMTO1 and MTO1 in VSMC treated with ox-LDL $(0,10,20,50 \mu \mathrm{g} / \mathrm{ml})$ for $24 \mathrm{~h}$. D The mRNA expression level of circMTO1 and MTO1 after treated with ox-LDL for different times $(0,12,24$ and $48 \mathrm{~h})$. ${ }^{*} P<0.05$, **P $<0.01$ and ${ }^{* * *} \mathrm{P}<0.001$

\section{CircMTO1 sponged and sequestered miR-182-5p to upregulate RASA1 expression}

Next, using Targetscan we predicted that RASA1 is a potential target for miR-182-5p (Fig. 4A). We found that miR-182-5p overexpression significantly reduced the luciferase activity of wild-type RASA1 vector $(\mathrm{P}<0.01)$, but not mutated RASA1 vector (Fig. 4B). Furthermore, luciferase activity of the wild-type RASA1 vector was also decreased by co-transfection with pcDNA-circMTO1 (wt) but not with pcDNA-circMTO1 with the mutated miR-182-5p binding site (Fig. 4B). In addition, compared with the miR-NC group, miR-182-5p overexpression significantly reduced the mRNA and protein expression of RASA1 $(\mathrm{P}<0.01)$, while circMTO1 overexpression significantly increased the mRNA and protein expression of RASA1 $(\mathrm{P}<0.01)$. Co-transfection of circMTO1 with miR-182-5p reversed the effect of miR-182-5p overexpression on RASA1 expression $(\mathrm{P}<0.05)$ (Fig. $4 \mathrm{C}$ and D). Besides, compared with the healthy group, RASA1 expression in AS patients was significantly reduced $(\mathrm{P}<0.01)$ (Fig. 4E). Furthermore, there was a significant positive correlation between circMTO1 and RASA1 expression in AS patients $(r=0.520, \mathrm{P}<0.001)$ (Fig. 4F).
These data showed that circMTO1 enhanced RASA1 expression by acting as a sponge for miR-182-5p in AS.

\section{CircMTO1 inhibited proliferation and migration of VSMCs} by regulating the miR-182-5p/RASA axis

To further confirm whether circMTO1 regulated AS progression via the miR-182-5p/RASA1 axis, the circMTO1 overexpression vector, miR-182-5p mimic, and siRNARASA1 were co-transfected into ox-LDL-stimulated VSMCs. As shown in Fig. 5A, B, compared with the NC group, circMTO1 overexpression significantly inhibited the viability and proliferation of VSMCs $(\mathrm{P}<0.05)$, while co-transfection with miR-182-5p or si-RASA1 significantly abolished the proliferation inhibition by circMTO1 overexpression $(\mathrm{P}<0.05)$. As shown in Fig. $5 \mathrm{C}$ and D, circMTO1 overexpression significantly induced VSMC apoptosis and inhibited VSMC migration, but these effects were abolished by co-transfection with miR-182-5p or si-RASA1 ( $<<0.05)$. Moreover, some protein markers critical to apoptosis and proliferation were also regulated by the circMTO $1 / \mathrm{miR}-182-5 \mathrm{p}-\mathrm{RASA} 1$ axis (Fig. 5E). These results indicated that miR-182-5p 


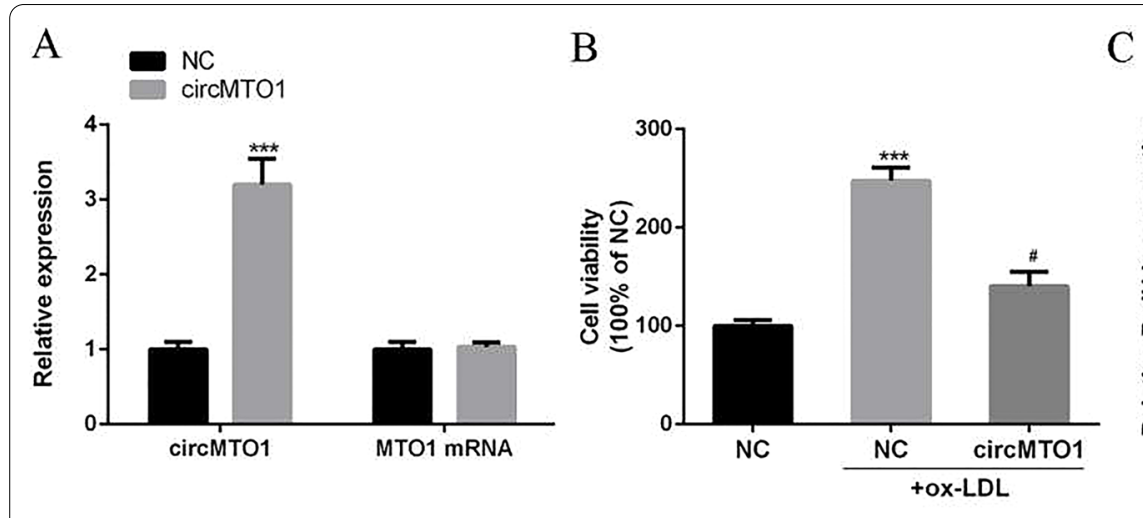

C

D
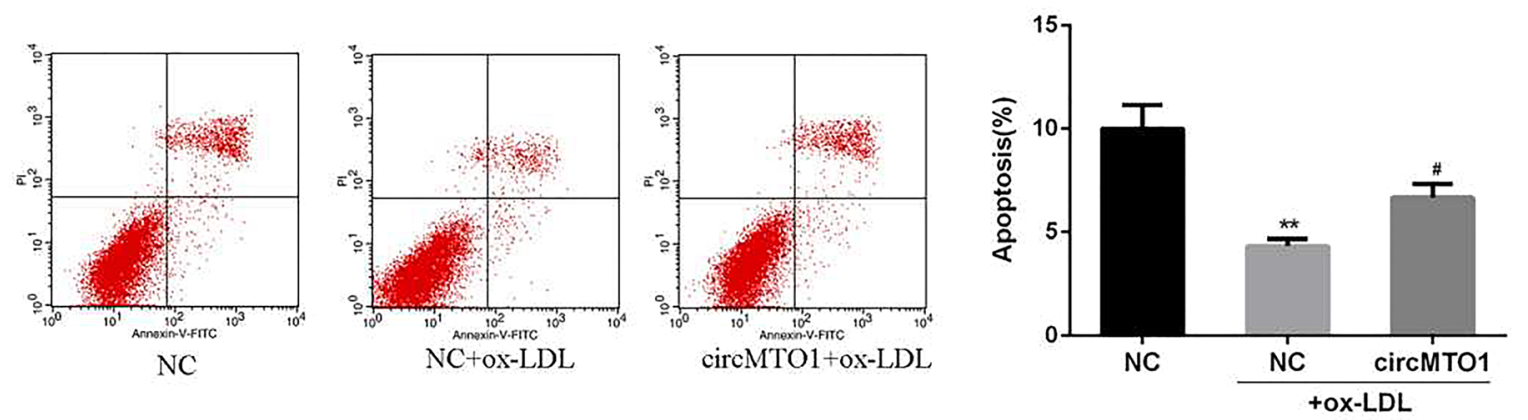

E

oh
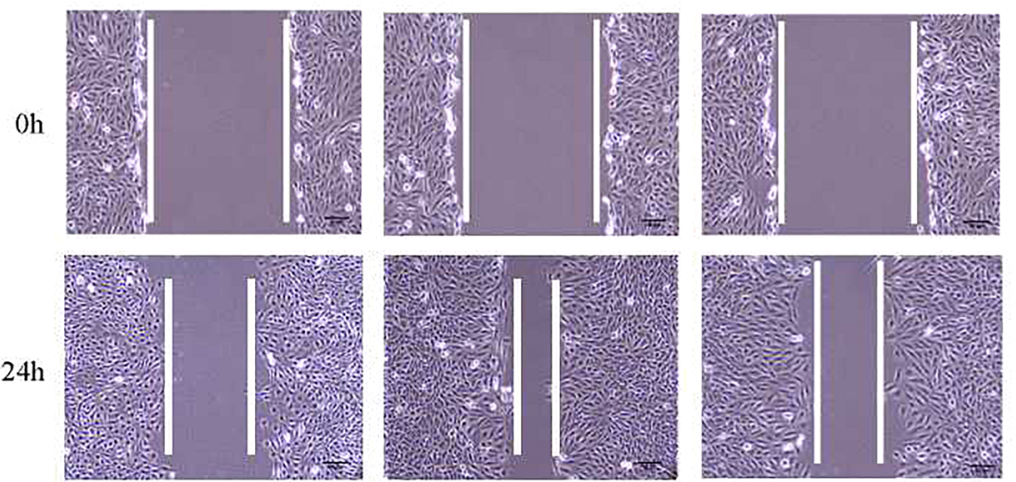

$\mathrm{NC}+\mathrm{Ca}$.

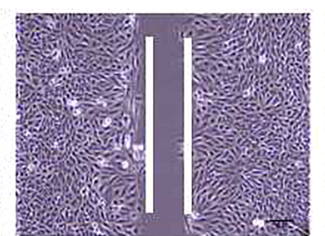

$\mathrm{NC}+$ ox-LDL $+\mathrm{Ca}$.

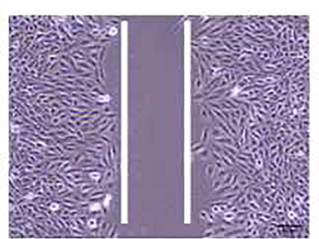

circMTO1+ox-LDL + Ca.

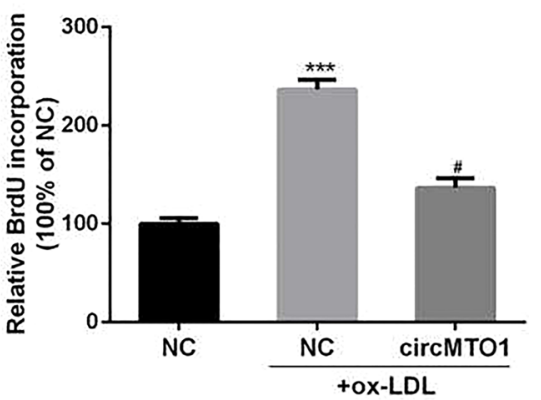

Fig. 2 CircMTO1 inhibited the proliferation and migration and induced the apoptosis of ox-LDL-stimulated VSMCs. A The mRNA expression levels of circMTO1 and MTO1 in VSMCs transfected with PCDNA 3.1-circMTO1. B, C The effect of circMTO1 overexpression on cell viability and proliferation determined by CCK8 and BrdU incorporation assays. D The effect of circMTO1 overexpression on apoptosis. $\mathbf{E}$ The effect of circMTO1 on cell migration. ${ }^{* *} \mathrm{P}<0.01,{ }^{\#} \mathrm{P}<0.05$ compared with $\mathrm{NC}+$ ox-LDL + capecitabine. Ca. indicates the capecitabine

overexpression or RASA1 knockdown reversed circMTO1-stimulated VSMC growth.

\section{circMTO1 overexpression attenuated arteriosclerosis by sponging miR-182-5p in vivo}

An AS mouse model was used to elucidate the function of the circMTO1/miR-182-5p axis, as described in the Materials and Method section, and AAV-pcDNA, AAV-pcDNA-circMTO1, and AAV-pcDNA-circMTO1/ AAV-miR-182-5p were injected into these mice. As shown in Fig. 6A, compared with the control group, the histological evaluation of oil-red and the immunochemistry staining of $\alpha$-SMA were both significantly increased in the AS group. circMTO1 overexpression significantly reduced the in situ staining of oil-red and $\alpha$-SMA (Fig. 6A). Co-injection of AAV-circMTO1 with AAVmiR-182-5p partially rescued the in situ oil-red staining trend of $\alpha$-SMA (Fig. 6A). RASA1 expression was also induced by injecting AAV-circMTO1 but attenuated by injecting AAV-miR-182-5p (Fig. 6B). The results indicated that circMTO1/miR-182-5p axis regulated the arteriosclerotic process. 

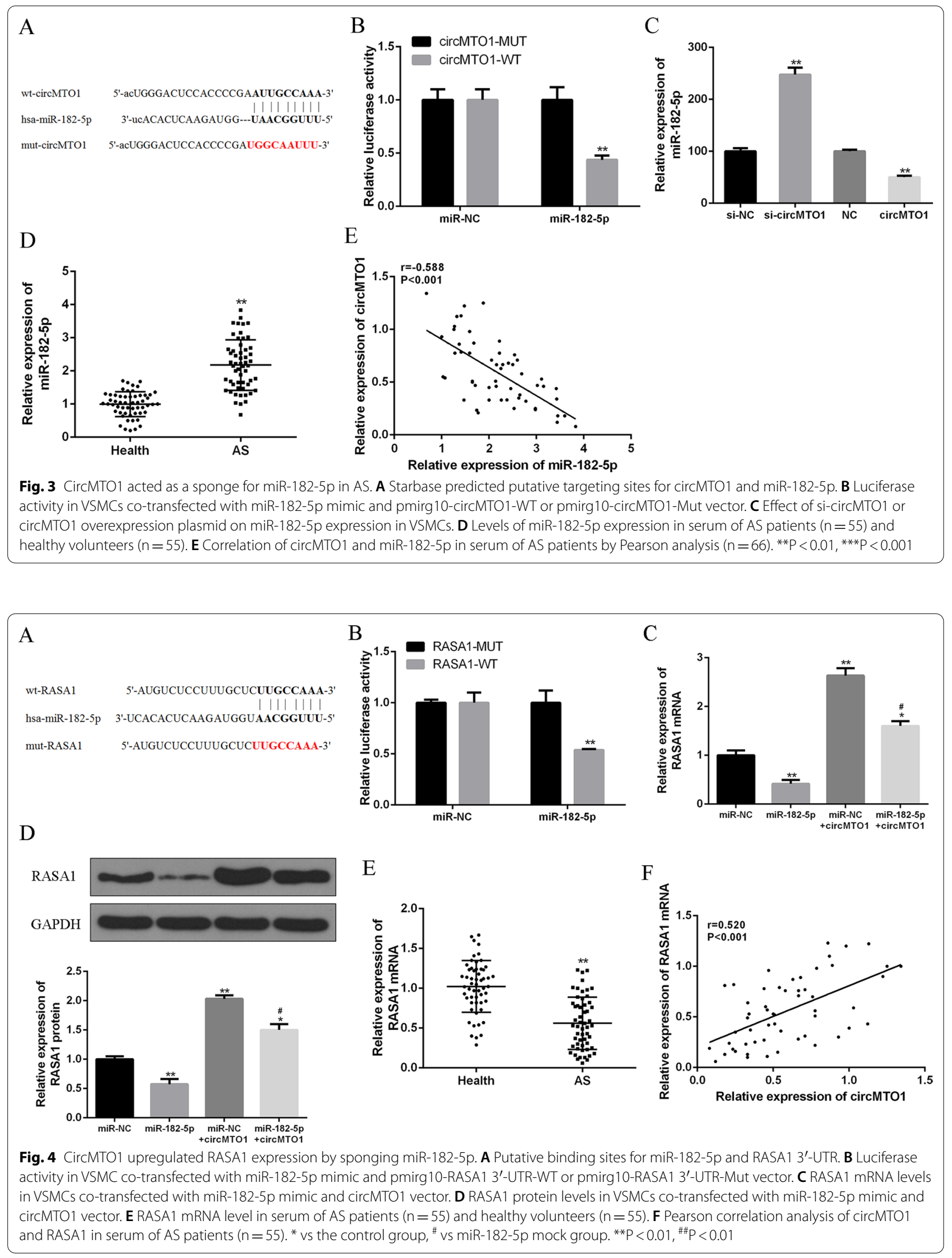


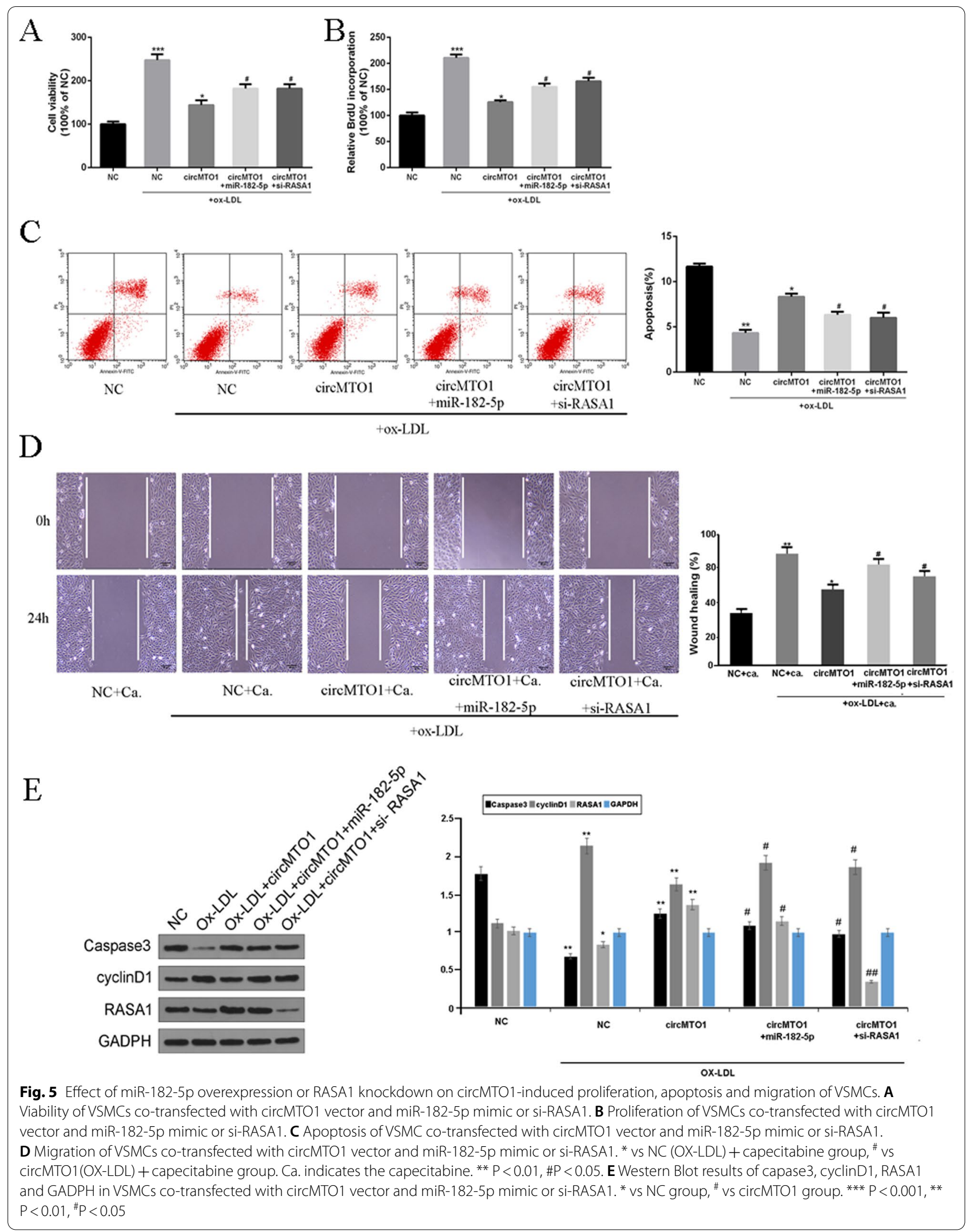




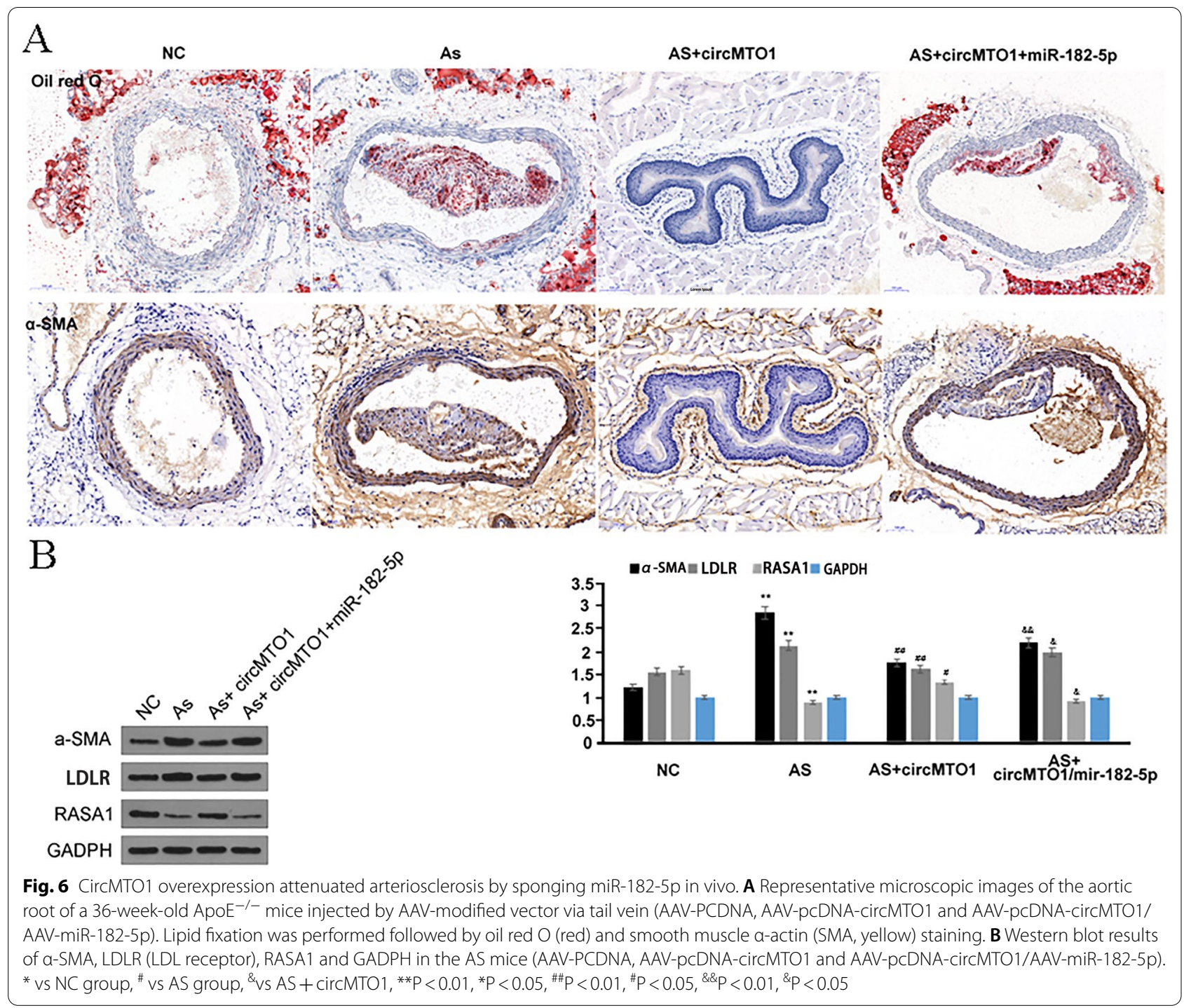

\section{Discussion}

Acute coronary events are common and severe diseases endangering human health (Wang et al. 2019b; Paigen et al. 1987). Various factors are involved in the pathogenesis and development of atherosclerosis. Among them, VSMCs play a critical role in intimal hyperplasia, plaque formation, and vascular stenosis (Arbabzadeh et al. 2012). VSMCs have high plasticity in terms of proliferation, migration, and transformation. When vascular injuries occur, VSMCs change from contractile type to synthetic type to promote blood vessels. However, under pathological conditions, the phenotype of VSMCs is dysregulated, which will lead to the development of cardiovascular diseases. Abnormal VSMC phenotype plays a critical role in atherosclerosis and other diseases after angioplasty (Zhao et al. 2014; Begum et al. 2011). Recently, it has been found that after vascular injury, some synthetic VSMCs are not derived from the transformation of contractile VSMCs but are derived from pluripotent vascular stem cells, suggesting that vascular wall-derived pluripotent stem cells differentiate into synthetic type VSMCs, which are important mechanisms for the development of atherosclerotic diseases (Mackay et al. 2011). The regulation mechanism in VSMC proliferation deserves further study.

With the deepening of basic research, circRNA has attracted more and more attention (Rubattu et al. 2014). In recent years, the critical regulatory role of circRNAs in cardiovascular diseases has gradually been recognized (Fan et al. 2017). For example, studies have found that circANRIL can bind to pescadillo homolog 1 (PES1), affecting pre-rRNA processing and ribosome production in 
VSMCs. CircANRIL can activate P53, induce apoptosis and inhibit proliferation(Nahapetyan et al. 2019). Studies have confirmed that circMTO1 plays a regulatory role in many diseases (Zhang et al. 2017). In glioblastoma, circMTO1 upregulation inhibits cell viability. In this study, we found that circMTO1 expression was decreased in AS patients and ox-LDL-stimulated VSMCs. Moreover, circMTO1 overexpression inhibited proliferation of ox-LDLstimulated VSMCs. Therefore, circMTO1 can regulate VSMC proliferation and migration.

As a target of circRNAs, miRNAs are the most widely studied noncoding RNAs, regulating cell proliferation and participating in the process of development, body metabolism, and tumorigenesis (Song et al. 2017). Many miRNAs have been shown to affect VSMC migration through different target genes or different pathways (Han et al. 2017b). For example, miR-145 directly targets PDCD4 to promote VSMC proliferation (Amin and Lam 2015). Recent studies have shown that miR-155 inhibits VSMC migration and proliferation via endothelial nitric oxide synthase (Han et al. 2017b). MiR-182-5p is differentially expressed in various diseases (Xue et al. 2016; Zhang et al. 2015) and predicted as a target gene for circMTO1. The miR-182-5p expression level was upregulated in AS patients and in VSMCs by circMTO1 knockdown. There was a negative correlation between the expression of circMTO1 and miR-182-5p. Co-transfection of miR-125b-5p with circMTO1 reversed the effect of circMTO1 on VSMC proliferation. These data indicated that circMTO1 regulates VSMC growth by modulating miR-182-5p.

RAS p21 protein activator 1 (RASA1) is one of the 14 RAS GTPases involving cell differentiation and apoptosis (Guo et al. 2016b). It can regulate cell proliferation and migration under the action of various growth factors such as PDGF and CSF-1 (Yao et al. 2016). Compared with other members, the role of RASA1 in diseases is poorly understood. RASA1 has a tumor suppressor effect in colorectal and pancreatic cancers, but its role in VSMCs is still unknown (Chenghuan et al. 2013). In this study, we found that RASA1 is a potential target for miR-182-5p. RASA1 mRNA level was decreased in AS patients, and circMTO1 expression was positively correlated with RASA1 level in the serum of AS patients. In VSMCs, RASA1 expression was significantly decreased in the miR-182-5p overexpression group but significantly increased in the circMTO1 overexpression group. Cotransfection of miR-125b-5p with circMTO1 reversed the effect of miR-182-5p on RASA1 expression. In addition, co-transfection of si-RASA1 with circMTO1 reversed the effect of circMTO1 on VSMC proliferation. These indicated that circMTO1 inhibits proliferation and enhances apoptosis by regulating miR-182-5p/RASA1 in ox-LDLstimulated VSMCs.

\section{Conclusion}

CircMTO1 inhibits proliferation and migration and induces apoptosis by regulating the miR-182-5p/RASA1 axis in ox-LDL-stimulated VSMCs and might be a potential therapeutic target for AS.

\section{Supplementary Information}

The online version contains supplementary material available at https://doi. org/10.1186/s10020-021-00330-2.

Additional file 1: The sequence of cirMTO1.

\section{Acknowledgements}

Not applicable.

\section{Authors' contributions}

All authors made substantial contributions to conception and design, data acquisition analysis and interpretation, manuscript drafting and revising, approved the final version to be published, and agreed to be accountable for all aspects of the work. All authors read and approved the final manuscript.

\section{Funding}

Jinhua Science and Technology Plan Project (2021-4-268), Jinhua Science and Technology Bureau (Grant Number 2019-4-138).

\section{Availability of data and materials}

The analyzed data sets generated during the study are available from the corresponding author on reasonable request.

\section{Declarations}

\section{Ethics approval and consent to participate}

The present study was approved by the Ethics Committee of Yiwu Central Hospital. The research has been carried out in accordance with the World Medical Association Declaration of Helsinki. All patients and healthy volunteers provided written informed consent prior to their inclusion within the study.

\section{Consent for publication}

Not applicable.

\section{Competing interests}

The authors declare no competing interests.

Received: 27 February 2020 Accepted: 16 June 2021

Published online: 08 July 2021

\footnotetext{
References

Amin M, Lam AK. Current perspectives of mi-RNA in oesophageal adenocarcinoma: roles in predicting carcinogenesis, progression and values in clinical management. Exp Mol Pathol. 2015;98(3):411-8.

Anokyedanso F, Trivedi CM, Juhr D, Gupta M, Cui Z, Tian Y, Zhang Y, Yang W, Gruber PJ, Epstein JA. Highly efficient miRNA-mediated reprogramming of mouse and human somatic cells to pluripotency. Cell Stem Cell. 2011;8(4):376-88.

Arbabzadeh A, Nakano M, Virmani R, Fuster V. Acute coronary events. Circulation. 2012;125(9):1147-56.

Asayama K, Hozawa A, Taguri M, Ohkubo T, Tabara Y, Suzuki K, Ando T, Harada A, Ohashi Y, Uechima H. Blood pressure, heart rate, and double product in
} 
a pooled cohort: the Japan Arteriosclerosis Longitudinal Study. J Hypertens. 2017;35(9):1808-15.

Ashwal-Fluss R, Meyer M, Pamudurti NR, Ivanov A, Bartok O, Hanan M, Evantal N, Memczak S, Rajewsky N, Kadener S. circRNA biogenesis competes with pre-mRNA splicing. Mol Cell. 2014;56(1):55-66.

Begum N, Hockman S, Manganiello VC. Phosphodiesterase 3A (PDE3A) deletion suppresses proliferation of cultured murine vascular smooth muscle cells (VSMCs) via inhibition of mitogen-activated protein kinase (MAPK) signaling and alterations in critical cell cycle regulatory proteins. J Biol Chem. 2011;286(29):26238-49.

Chen X, Wu QF, Yan GY. RKNNMDA: ranking-based KNN for MiRNA-disease association prediction. RNA Biol. 2017;14(7):1-11.

Chenghuan HU, Huang F, Deng G, Nie W, Huang W, Zeng XI. miR-31 promotes oncogenesis in intrahepatic cholangiocarcinoma cells via the direct suppression of RASA1. Exp Ther Med. 2013;6(5):1265-70.

Fan X, Weng X, Zhao Y, Chen W, Gan T, Xu D. Circular RNAs in cardiovascular disease: an overview. Biomed Res Int. 2017;2017(6):5135781.

Gong B, Liu WW, Nie WJ, Li DF, Xie ZJ, Liu C, Liu YH, Mei P, Li ZJ. MiR-21/RASA1 axis affects malignancy of colon cancer cells via RAS pathways. World J Gastroenterol. 2015;21(5):1488-97.

Gruner H, Cortéslópez M, Cooper DA, Bauer M, Miura P. CircRNA accumulation in the aging mouse brain. Sci Rep. 2016;6(1):38907.

Guo X, Li D, Chen M, Chen L, Zhang B, WU T, Guo R. miRNA-145 inhibits VSMC proliferation by targeting CD40. Sci Rep. 2016a;6:35302.

Guo X, Yu L, Chen M, Wu T, Peng X, Guo R, Zhang B. miR-145 mediated the role of aspirin in resisting VSMCs proliferation and anti-inflammation through CD40. J Transl Med. 2016b;14(1):1-10.

Han D, Li J, Wang H, Su X, Hou J, Gu Y, Qian C, Lin Y, Liu X, Huang M. Circular RNA circMTO1 acts as the sponge of microRNA-9 to suppress hepatocellular carcinoma progression. Hepatology. 2017a;66(4):1151.

Han D, Li J, Wang H, Su X, Hou J, Gu Y, Qian C, Lin Y, Liu X, Huang M. Circular RNA MTO1 acts as the sponge of miR-9 to suppress hepatocellular carcinoma progression. Hepatology. 2017b;66(4):1151.

Hancock ML, Preitner N, Quan J, Flanagan JG. MicroRNA-132 is enriched in developing axons, locally regulates Rasa1 mRNA, and promotes axon extension. J Neurosci. 2014;34(1):66-78.

Holdt LM, Kohlmaier A, Teupser D. Molecular functions and specific roles of circRNAs in the cardiovascular system. Non-Coding RNA Res. 2018;3(2):75-98.

Jin X, Feng C, Xiang Z, Chen Y, Li Y. CircRNA expression pattern and circRNAmiRNA-mRNA network in the pathogenesis of nonalcoholic steatohepatitis. Oncotarget. 2016;7(41):66455-67.

Kuhar MJ, Yoho LL. CART peptide analysis by Western blotting. Synapse. 2015;33(3):163-71.

Li R, Wen X, Wang S, Wu Q, Yang N, Xin Y, Pan H, Zhou X, Bai L, Xu B. Reference gene selection for qRT-PCR analysis in the Sweetpotato Whitefly, Bemisia tabaci (Hemiptera: Aleyrodidae). PLoS ONE. 2013;8(1):e53006.

Li C, Du X, Tai S, Zhong X, Wang Z, Hu Z, Zhang L, Kang P, Ji D, Jiang X. GPC1 regulated by miR-96-5p, rather than miR-182-5p, in inhibition of pancreatic carcinoma cell proliferation. Int J Mol Sci. 2014;15(4):6314-27.

Li W, Zhi W, Liu F, He Z, Wang X, Niu X. Atractylenolide I restores HO-1 expression and inhibits Ox-LDL-induced VSMCs proliferation, migration and inflammatory responses in vitro. Exp Cell Res. 2017:353(1):26-34.

Li L, Mao D, Li C, Li M. miR-145-5p inhibits vascular smooth muscle cells (VSMCs) proliferation and migration by dysregulating the transforming growth factor- $\beta$ signaling cascade. Med Sci Monit. 2018;24:4894-904.

Lippincott WW. Arteriosclerosis, thrombosis, and vascular biology. Arterioscler Thromb Vasc Biol. 2017;37(1):1.

Mackay DF, Irfan MO, Haw S, Pell JP. Meta-analysis of the effect of comprehensive smoke-free legislation on acute coronary events. Postgrad Med J. 2011;87(1026):311.

Nahapetyan H, Moulis M, Grousset E, Faccini J, Grazide MH, Mucher E, Elbaz M, Martinet W, Vindis C. Altered mitochondrial quality control in Atg7deficient VSMCs promotes enhanced apoptosis and is linked to unstable atherosclerotic plaque phenotype. Cell Death Dis. 2019;10(2):119.
Paigen B, Morrow A, Holmes PA, Mitchell D, Williams RA. Quantitative assessment of atherosclerotic lesions in mice. Atherosclerosis. 1987;68:231-40.

Piwecka M, Glažar P, Hernandez-Miranda LR, Memczak S, Wolf SA, Rybak-Wolf A, Filipchyk A, Klironomos F, Cerda Jara CA, Fenske P. Loss of a mammalian circular RNA locus causes miRNA deregulation and affects brain function. Science. 2017;357(6357):eaam8526.

Qu S, Zhong Y, Shang R, Zhang X, Song W, Kjems J, Li H. The emerging landscape of circular RNA in life processes. RNA Biol. 2017;14(8):992-9.

Revencu N, Boon LM, Mendola A, Cordisco MR, Dubois J, Clapuyt P, Hammer F, Amor DJ, Irvine AD, Baselga E. RASA1 mutations and associated phenotypes in 68 families with capillary malformation-arteriovenous malformation. Hum Mutat. 2013;34(12):1632-41.

Rotllan N, Wanschel AC, Fernandezhernando A, Salerno AG, Offermanns S, Sessa WC, Fernándezhernando C. Genetic evidence supports a major role for Akt1 in VSMCs during atherogenesis. Circ Res. 2015;116(11):1744-52.

Rubattu S, Marchitti S, Bianchi F, Di CS, Stanzione R, Cotugno M, Bozzao C, Sciarretta S, Volpe M. The C2238/aANP variant is a negative modulator of both viability and function of coronary artery smooth muscle cells. PLoS ONE. 2014;9(11):e113108.

Song CL, Wang JP, Xue X, Liu N, Zhang XH, Zhao Z, Liu JG, Zhang CP, Piao ZH, Liu Y. Effect of circular ANRIL on the inflammatory response of vascular endothelial cells in a rat model of coronary atherosclerosis. Cell Physiol Biochem. 2017:42(3):1202-12

Tabib A, Leroux C, Mornex JF, Loire R. Accelerated coronary atherosclerosis and arteriosclerosis in young human-immunodeficiency-virus-positive patients. Coron Artery Dis. 2017;11(1):41.

Tölle M, Reshetnik A, Schuchardt M, Höhne M, Giet M. Arteriosclerosis and vascular calcification: causes, clinical assessment and therapy. Eur J Clin Invest. 2015:45(9):976-85.

Wallace C, Newhouse SJ, Braund P, Zhang F, Tobin M, Falchi M, Ahmadi K, Dobson RJ, Marçano ACB, Hajat C. Genome-wide association study identifies genes for biomarkers of cardiovascular disease: serum urate and dyslipidemia. Am J Hum Genet. 2008;82(1):139-49.

Wang M, Wu Y, Yu Y, Fu Y, Yan H, Wang X, Li T, Peng W, Luo D. Rutaecarpine prevented ox-LDL-induced VSMCs dysfunction through inhibiting overexpression of connexin 43. Eur J Pharmacol. 2019a;853:84-92.

Wang Y, Dubland JA, Allahverdian S, et al. Smooth muscle cells contribute the majority of foam cells in ApoE (Apolipoprotein E)-deficient mouse atherosclerosis. Arterioscler Thromb Vasc Biol. 2019b;39(5):876-87.

Xue J, Zhou A, Wu Y, Morris SA, Lin K, Amin S, Verhaak R, Fuller G, Xie K, Heimberger AB. miR-182-5p induced by STAT3 activation promotes glioma tumorigenesis. Can Res. 2016;76(14):4293.

Yao J, Xu C, Fang Z, Li Y, Liu H, Wang Y, Xu C, Sun Y. Androgen receptor regulated microRNA miR-182-5p promotes prostate cancer progression by targeting the ARRDC3/ITGB4 pathway. Biochem Biophys Res Commun. 2016;474(1):213-9.

Zhang HB, Wen JK, Zhang J, Miao SB, Ma GY, Wang YY, Zheng B, Han M. Flavonoids from Inula britannica reduces oxidative stress through inhibiting expression and phosphorylation of p47phox in VSMCs. Pharm Biol. 2011:49(8):815.

Zhang J, Zhao F, Yu X, Lu X, Zheng G. MicroRNA-155 modulates the proliferation of vascular smooth muscle cells by targeting endothelial nitric oxide synthase. Int J Mol Med. 2015;35(6):1708.

Zhang HD, Jiang LH, Sun DW, Hou JC, Ji ZL. CircRNA: a novel type of biomarker for cancer. Breast Cancer. 2017;25(1):1-7.

Zhao Y, Feng G, Wang Y, Yue Y, Zhao W. Regulation of apoptosis by long noncoding RNA HIF1A-AS1 in VSMCs: implications for TAA pathogenesis. Int J Clin Exp Pathol. 2014;7(11):7643.

\section{Publisher's Note}

Springer Nature remains neutral with regard to jurisdictional claims in published maps and institutional affiliations. 